\title{
Polarization of Lyman $\alpha$ Emergent from a Thick Slab of Neutral Hydrogen
}

\author{
Sang-Hyeon Ahn ${ }^{1}$ And Hee-Won LeE ${ }^{2}$ \\ ${ }^{1}$ Korea Astronomy and Space Science Institute, Daedeok-daero 776, Yuseong-gu, Daejeon 305-348, Korea; sha@kasi.re.kr \\ ${ }^{2}$ Department of Astronomy and Space Science, Sejong University, Neungdong-ro 209, Gwangjin-gu, Seoul 143-747, Korea \\ hwlee@sejong.ac.kr
}

Received October 27, 2014; accepted June 11, 2015

\begin{abstract}
Star forming galaxies found in the early universe exhibit asymmetric Ly $\alpha$ emission line that results from multiple scattering in a neutral thick medium surrounding the Ly $\alpha$ emission source. It is expected that emergent Ly $\alpha$ will be significantly polarized through a large number of resonance scattering events followed by a number of successive wing scatterings. In this study we adopt a Monte Carlo method to calculate the polarization of Ly $\alpha$ transferred in a very thick static slab of H I. Resonantly scattered radiation associated with transitions between $1 S_{\frac{1}{2}}-2 P_{\frac{1}{2}, \frac{3}{2}}$ is only weakly polarized and therefore linear polarization of the emergent Ly $\alpha$ is mainly dependent on the number of off-resonant wing scattering events. The number of wing scattering events just before escape from the slab is determined by the product of the Doppler parameter $a$ and the line center optical depth $\tau_{0}$, which, in turn, determines the behavior of the linear polarization of $\operatorname{Ly} \alpha$. This result is analogous to the study of polarized radiative transfer of Thomson scattered photons in an electron slab, where the emergent photons are polarized in the direction perpendicular to the slab when the scattering optical depth is small and polarized in the parallel direction when the slab is optically thick. Our simulated spectropolarimetry of Ly $\alpha$ shows that the line center is negligibly polarized, the near wing parts polarized in the direction parallel to the slab and the far wing parts are polarized in the direction perpendicular to the slab. We emphasize that the flip of polarization direction in the wing parts of Ly $\alpha$ naturally reflects the diffusive nature of the Ly $\alpha$ transfer process in thick neutral media.
\end{abstract}

Key words: profile: radiative transfer: polarization

\section{INTRODUCTION}

Cosmic reionization is an outstanding event in the history of the universe driven by the strong UV radiation from the first objects in the early Universe. Galaxies with high redshifts $z>5$ exhibit a Ly $\alpha$ emission line with an asymmetric profile where the blue part is significantly suppressed. This characteristic profile of $\operatorname{Ly} \alpha$ is indicative of multiple scattering in an expanding neutral medium, where escape is allowed to only those photons whose frequencies are shifted significantly redward via either resonance or wing scattering with hydrogen atoms in motion (Ahn et al. 2003). Starburst galaxies at high redshifts exhibit a Ly $\alpha$ halo with a half-light radius of about $1 \mathrm{kpc}$ (Bouwens et al. 2004). The Ly $\alpha$ halo appears to be formed by multiply scattered Ly $\alpha$ photons that originate in the central star forming region.

Ly $\alpha$ is a resonance doublet arising from $1 S_{\frac{1}{2}} \rightarrow 2 P_{\frac{1}{2}, \frac{3}{2}}$ transitions. The scattering optical depth of Ly $\alpha$ emergent from star forming galaxies can be very large near the line center, requiring a careful treatment of the radiative transfer (Ahn et al. 2001, 2002). The transfer of Ly $\alpha$ in a very thick neutral medium is regarded as a diffusion process occurring both in frequency space and real space (Neufeld 1990). We will consider in this

CORRESPONDING AUthor: S. Ahn study the radiative transfer of Ly $\alpha$ photons having frequency near the line center frequencies corresponding to the doublet transitions $2 P \rightarrow 1 S$. In this case, the scattering is resonant, which is quite local in real space due to the enormous scattering optical depth at the line center. However, a large number of resonant scattering events eventually push the Ly $\alpha$ photon out of line center in the frequency space. The photon gets redistributed to the wing parts where the scattering optical depth is quite small. Spatial excursion becomes more important at this point and the scattering geometry may affect the final polarization state of emergent photons.

Polarimetry plays an important role in revealing essential information regarding the transfer process of Ly $\alpha$ in this environment. The polarization of resonantly scattered line photons is determined by the quantum numbers of lower and upper states in addition to the relative orientation of the incident radiation and outgoing photon. In particular in the case of the $J=\frac{1}{2} \rightarrow \frac{1}{2}$ transition, the scattered radiation is completely isotropic and unpolarized irrespective of the scattering angle between the incident and scattered radiation. For the $J=\frac{1}{2} \rightarrow \frac{3}{2}$ transition, the linear degree of polarization is $\frac{3}{7}$ for a right angle scattering when the incident radiation is unpolarized. A right angle scattering for a $J=0 \rightarrow 1$ transition yields completely linearly polarized radiation. Far off resonance, 
a complete linear polarization is obtained, which is regarded as Rayleigh scattering. In general, the linear polarization of a line photon scattered in the near wing part is dependent on the wavelength due to quantum interference between the two transitions $S_{\frac{1}{2}}$ and $P_{\frac{1}{2}, \frac{3}{2}}$ (e.g., Stenflo 1980, 1994, 1996; Lee et al. 1994).

However, in the case of $\operatorname{Ly} \alpha$, the energy difference between the two fine structure levels is very small, so that the quantum interference effects in the near wing parts can be safely neglected. Therefore in this work we will only consider the three cases, resonance scattering with $S_{\frac{1}{2}}-P_{\frac{1}{2}}$, resonance scattering with $S_{\frac{1}{2}}-P_{\frac{3}{2}}$ and off-resonance scattering. In this respect, Ly $\alpha$ is quite interesting with regard to polarization, because a slight change in frequency in the rest frame of the scattering hydrogen atom results in entirely different polarization in the scattered photon.

When a Ly $\alpha$ emitting source is surrounded by a geometrically thin and optically thick neutral shell, we expect that the Ly $\alpha$ scattered in the shell can be polarized depending on the number of wing scatterings. The linear polarization of the continuum radiation transferred in an optically thick shell consisting of free electrons was investigated by Chandrasekhar (1960), who showed that up to 11.7 percent of linear polarization is obtained for radiation emergent in the grazing direction of the plane. Phillips \& Mészáros (1986) found that the linear polarization is varied depending on the optical thickness of the electron scattering medium. They showed that a thin shell is characterized by linear polarization in the direction perpendicular to the electron scattering plane, whereas in the case of a thick shell the linear polarization develops in the direction parallel to the plane.

Ly $\alpha$ photons emerging from a very thick neutral shell will be polarized in a way similar to the Thomson scattered radiation in the regime dominated by wing scattering following a sufficient number of resonant scatterings. It was pointed out that $\operatorname{Ly} \alpha$ will be polarized in a neutral medium when the medium takes the form of a geometrically thin shell (Lee \& Ahn 1998). The polarimetric observation of a Ly $\alpha$ blob by Hayes et al. (2011) shows that Ly $\alpha$ from early universe is indeed polarized significantly.

In this paper, we investigate the behavior of the linear polarization that Ly $\alpha$ photons acquire during their transfer in a very thick slab of neutral hydrogen where escape is possible only after sufficient diffusion in the frequency space.

\section{MODEL}

\subsection{Radiative Transfer in a Very Thick Medium}

The neutral hydrogen column density $N_{\mathrm{HI}}$ of the Ly $\alpha$ halo around a starburst galaxy is inferred to be in the range $N_{H I}=10^{18}-10^{22} \mathrm{~cm}^{-2}$ (e.g., Kunth et al. 1998; Pettini et al. 2001). A typical line center optical depth of a resonance line is approximately related to $N_{\mathrm{HI}}$ by

$$
\tau_{0}=5.89 \times 10^{6} T_{4}^{-1 / 2}\left[\frac{N_{\mathrm{HI}}}{10^{20} \mathrm{~cm}^{-2}}\right],
$$

where $T_{4}$ is the temperature of the medium in units of $10^{4} \mathrm{~K}$ and $N_{\mathrm{HI}}$ is the column density of the atomic or ionic species ${ }^{\mathbf{1}}$. Extremely large optical depths at line center ranging $\tau_{0}=10^{6}-10^{9}$ characterize the neutral region surrounding the $\operatorname{Ly} \alpha$ emission source found in the early universe.

In a moderately optically thick medium with $\tau_{0} \simeq$ $10^{4}$, the escape of resonantly scattered photons is made through a small number of wing scatterings. In a very thick medium, wing scattering plays a critical role in the transfer of resonance line photons, which is similar to a diffusion process. Previous researchers (Avery \& House 1968; Adams 1972; Harrington 1973; Neufeld 1990) introduced the diffusion approximation where only wing scatterings take place during the transfer. This approximation is quite good for the extremely thick cases with $a \tau_{0}>10^{3}$, where $a$ is the Voigt parameter given by $a=4.71 \times 10^{-4} T_{4}^{-1 / 2}$.

However, Ly $\alpha$ photons experience both core scatterings and wing scatterings and may alternate between these two types of scattering during the line radiative transfer (Ahn et al. 2000). A large number of core scatterings change the polarization vector in a random way to yield an isotropic and unpolarized radiation field. Therefore, both wing scatterings and core scatterings should be fully considered in order to compute accurately the polarization of $\mathrm{Ly} \alpha$ emerging from a very thick H I medium.

We denote by $\nu_{\frac{1}{2}}$ and $\nu_{\frac{3}{2}}$ the resonance frequencies for the transitions $1 S_{\frac{1}{2}}-2 P_{\frac{1}{2}}$ and $1 S_{\frac{1}{2}}-2 P_{\frac{3}{2}}$, respectively. The level-splitting $\Delta \nu_{P}=\nu_{\frac{3}{2}}-\nu_{\frac{1}{2}}=10 \mathrm{GHz}$ is very small, amounting to the Doppler shift of $1.34 \mathrm{~km} \mathrm{~s}^{-1}$ relative to the average of Ly $\alpha$ line center. In the rest frame of a scattering hydrogen atom, the cross section of an incident Ly $\alpha$ photon with frequency $\nu$ is excellently approximated by a combination of Lorentzian $\phi_{L}(\nu)$ functions given by

$$
\begin{aligned}
\phi_{L}(\nu) & =\frac{1}{3}\left[\frac{\Gamma / 4 \pi^{2}}{\left(\nu-\nu_{\frac{1}{2}}\right)^{2}+(\Gamma / 4 \pi)^{2}}\right] \\
& +\frac{2}{3}\left[\frac{\Gamma / 4 \pi^{2}}{\left(\nu-\nu_{\frac{3}{2}}\right)^{2}+(\Gamma / 4 \pi)^{2}}\right],
\end{aligned}
$$

where $\Gamma=6.25 \times 10^{8} \mathrm{~s}^{-1}$ is the radiative damping parameter.

The line profile function of a resonance line from a thermal velocity distribution with temperature $T$ is described by the Voigt function $H(a, x)$, where

$$
H(a, x) \equiv \frac{a}{\pi} \int_{-\infty}^{\infty} \frac{e^{-y^{2}} d y}{a^{2}+(x-y)^{2}},
$$

\footnotetext{
${ }^{1}$ We note that Equation (4) in Ahn et al. (2000), Equation (3) in Ahn et al. (2001), Equation (8) in Ahn et al. (2002), and Equation (1) in Ahn et al. (2003) are in error.
} 
where $x$ is the dimensionless frequency deviation from the line center $x \equiv\left(\nu-\nu_{0}\right) / \Delta \nu_{D}$ with the Doppler width $\Delta \nu_{D} \equiv \nu_{0}\left(v_{t h} / c\right)$. Here, $\nu_{0}$ and $v_{t h}$ are the line center frequency and the thermal speed, respectively. The Voigt parameter $a \equiv \Gamma /\left(4 \pi \Delta \nu_{D}\right)$ measures the natural width in terms of the Doppler width.

In the case of Ly $\alpha$, for which the two resonance doublets are located closely with each other in frequency space, the line profile function is given by the sum of two Voigt functions

$$
\phi_{\mathrm{Ly} \alpha}(\nu)=\frac{H\left(a, x_{1}\right)+2 H\left(a, x_{2}\right)}{3 \Delta \nu_{D} \sqrt{\pi}},
$$

where $x_{1} \equiv\left(\nu-\nu_{\frac{1}{2}}\right) / \Delta \nu_{D}$ and $x_{2} \equiv\left(\nu-\nu_{\frac{3}{2}}\right) / \Delta \nu_{D}$.

Because the level-splitting is much smaller than the thermal speed of a medium with $T \geq 100 \mathrm{~K}$, one normally neglects the $2 P_{\frac{1}{2}}, \frac{3}{2}$ level-splitting, treating it as a single level. Even when $T \leq 100 \mathrm{~K}$, the level-splitting effects may also be neglected in the cases where the line center optical depth of the scattering medium is very large. However, the polarization of the scattered Ly $\alpha$ is sensitively dependent on the scattering type, and therefore it is important to distinguish the scattering type during the transfer of Ly $\alpha$ photons.

Given frequency of the incident radiation, the scattering type is determined to be one out of three possibilities. More specifically, an isotropic and completely unpolarized radiation results from a transition $1 S_{\frac{1}{2}}-2 P_{\frac{1}{2}}$. On the other hand, weakly polarized radiation field is obtained from a transition $1 S_{\frac{1}{2}}-2 P_{\frac{3}{2}}$, where a maximum degree of linear polarization of $3 / 7$ is obtained when an outgoing photon propagates perpendicularly to the direction of incidence. Due to the small level splitting of $2 P_{\frac{1}{2}}$ and $2 P_{\frac{3}{2}}$ of atomic hydrogen, a particulary interesting case is obtained for a wing scattering or off-resonance scattering of $\operatorname{Ly} \alpha$, for which the polarization behavior is the same as that of the classical Rayleigh scattering.

According to Ahn et al. (2001), the probability $P_{r 1}$ that a Ly $\alpha$ photon is scattered resonantly with the transition $1 S_{\frac{1}{2}}-2 P_{\frac{1}{2}}$ is given by

$$
P_{r 1} \simeq e^{-x_{1}^{2}} /\left[H\left(a, x_{1}\right)+2 H\left(a, x_{2}\right)\right],
$$

whereas the probability $P_{r 2}$ for resonant scattering with $1 S_{\frac{1}{2}}-2 P_{\frac{3}{2}}$ is given by

$$
P_{r 2} \simeq 2 e^{-x_{2}^{2}} /\left[H\left(a, x_{1}\right)+2 H\left(a, x_{2}\right)\right] .
$$

The probability that a given scattering is off-resonant is therefore given by

$$
P_{n r}=1-\left(P_{r 1}+P_{r 2}\right) .
$$

A Monte Carlo code was developed by Ahn et al. $(2000,2001,2002)$ to deal with the Ly $\alpha$ line transfer in optically thick media. When the medium is moderately optically thick, the photon escape is made by a couple of wing scatterings after a significant number of

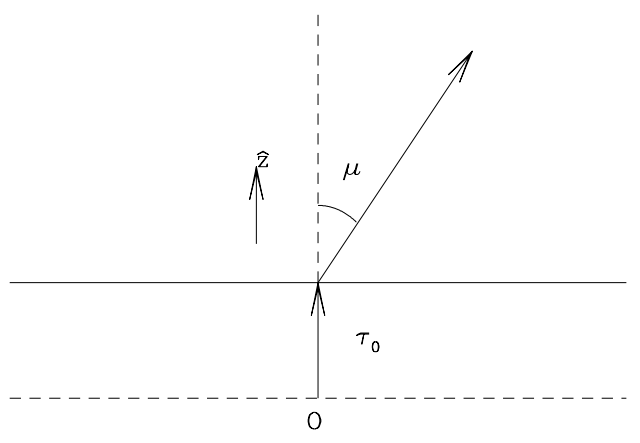

Figure 1. Model configuration adopted in this work. We consider a static and uniform slab with a $\operatorname{Ly} \alpha$ source at the origin denoted in the figure by $\mathrm{O}$. The vertical thickness of the slab is $2 \tau_{0}$, and $\mu$ is the cosine of the angle between the wave vector of the emergent photon and the slab normal.

core scatterings. In this case, due to a small number of wing scatterings the diffusion approximation is not reliable. In the case of extremely thick medium, emergent photons experience a significant number of successive wing scatterings just before escape, rendering the diffusion approximation valid. The Monte Carlo code accurately treats the partial frequency redistribution. In other words, the frequency redistribution comes from the Doppler redistribution when the resonant scattering occurs in the rest frame of the atom. For each scattering event, the scattering type between resonant core and non-resonant wing scattering, is determined in a probabilistic way depending on the frequency of the photon along its propagation direction.

The small level-splitting lying in the transitions is in most cases neglected when one is interested only in the total line flux. However, in the case of polarized radiative transfer, the linear polarization differs greatly depending on the scattering type. Hence, although we adopted the same Monte Carlo code developed by Ahn et al. (2000, 2001, 2002), the Ly $\alpha$ line transfer is treated in a manner very faithful to the atomic physics taking full considerations of the fine structure of atomic hydrogen.

\subsection{Density Matrix Formalism for Scattering of $\operatorname{Ly} \alpha$}

In Figure 1 we show an illustration of the scattering medium considered in this work. The scattering medium takes a form of a slab with a finite thickness consisting of neutral hydrogen. The lateral extent of the slab is considered to be infinite and we take $z$-axis to be the normal direction of the slab.

In the Monte Carlo code, each photon is followed until its escape from the medium, keeping the wavevector and the density matrix associated with the photon. The wave vector is given in the spherical coordinate in the rest frame of a hydrogen atom, in which the polar angle is denoted by $\theta$ and the azimuth angle is given by $\phi$. For the unit wavevector of the incident Ly $\alpha$ denoted 
by $\hat{\mathbf{k}}=(\sin \theta \cos \phi, \sin \theta \sin \phi, \cos \theta)$, we may choose the two polarization basis vectors

$$
\begin{aligned}
\epsilon_{1} & =(-\sin \phi, \cos \phi, 0) \\
\epsilon_{2} & =(\cos \theta \cos \phi, \cos \theta \sin \phi,-\sin \theta) .
\end{aligned}
$$

With this convention, $\epsilon_{1}$ represents polarization perpendicular to the symmetry axis, which is taken to coincide with the $z$-axis. The polarization in the direction of the plane spanned by $\hat{\mathbf{k}}$ and $\hat{\mathbf{z}}$ is represented by $\epsilon_{2}$. By primed quantities we denote the corresponding quantities for the wave vector of the scattered Ly $\alpha$. For example, $\hat{\mathbf{k}}^{\prime}=\left(\sin \theta^{\prime} \cos \phi^{\prime}, \sin \theta^{\prime} \sin \phi^{\prime}, \cos \theta^{\prime}\right)$ is the unit wavevector for the scattered Ly $\alpha$.

We adopt the density operator formalism to characterize the statistically mixed state of Ly $\alpha$ photons in our Monte Carlo code (e.g., Lee et al. 1994). In this formalism the $2 \times 2$ Hermitian density matrix $\rho$ is related to the conventional Stokes parameters $I, Q, U$ and $V$ by

$$
\begin{aligned}
& \rho_{11}=(I+Q) / 2 \\
& \rho_{22}=(I-Q) / 2 \\
& \rho_{12}=(U+i V) / 2 .
\end{aligned}
$$

In this work, the density matrix is normalized by the condition that it has a unit trace. The calculation of a Ly $\alpha$ photon transfer in the Monte Carlo simulation begins with an unpolarized photon that is described by the density matrix

$$
\rho=\frac{1}{2}\left(\begin{array}{ll}
1 & 0 \\
0 & 1
\end{array}\right) .
$$

If the scattering is resonant with the transition $1 S_{\frac{1}{2}}-$ $2 P_{\frac{1}{2}}$, Ly $\alpha$ is isotropically scattered and completely unpolorized with $\rho$ given by Equation (10) irrespective of the wavevector of the incident Ly $\alpha$. If the scattering is resonant with the transition $S_{\frac{1}{2}}-P_{\frac{3}{2}}$ the density matrix associated with the scattered Ly $\alpha$ is given in the Equation (5) of Ahn et al. (2002). However, for the clarification we arrange the terms and write the equation as follows,

$$
\begin{aligned}
\rho_{11}^{\prime}= & (5+3 \cos 2 \Delta \phi) \rho_{11} \\
+ & {\left[(5-3 \cos 2 \Delta \phi) \cos ^{2} \theta+2 \sin ^{2} \theta\right] \rho_{22} } \\
- & (6 \cos \theta \sin 2 \Delta \phi) \rho_{12} \\
\rho_{12}^{\prime}= & \left(3 \sin 2 \Delta \phi \cos \theta^{\prime}\right) \rho_{11} \\
+ & 6\left(\cos \theta \cos \theta^{\prime} \cos 2 \Delta \phi+\sin \theta \sin \theta^{\prime} \cos \Delta \phi\right) \rho_{12} \\
+ & 3 \cos \theta\left(-2 \sin \theta \sin \theta^{\prime} \sin \Delta \phi\right. \\
& \left.-\cos \theta \cos \theta^{\prime} \sin 2 \Delta \phi\right) \rho_{22} \\
= & {\left[(5-3 \cos 2 \Delta \phi) \cos ^{2} \theta^{\prime}+2 \sin ^{2} \theta^{\prime}\right] \rho_{11} } \\
\rho_{22}^{\prime} & {\left[(5+3 \cos 2 \Delta \phi) \cos ^{2} \theta \cos ^{2} \theta^{\prime}\right.} \\
& +2 \cos { }^{2} \theta \sin ^{2} \theta^{\prime} \\
& +12 \cos \Delta \phi \cos \theta \cos \theta^{\prime} \sin \theta \sin ^{\prime} \\
& \left.\left.+2 \cos \theta^{2} \sin ^{2} \theta+8 \sin ^{2} \theta \sin ^{2} \theta^{\prime}\right)\right] \rho_{22} \\
+ & \left(6 \sin 2 \Delta \phi \cos ^{2} \theta^{\prime} \cos \theta\right. \\
& \left.+2 \sin \Delta \phi \cos ^{\prime} \sin \theta \sin ^{\prime}\right) \rho_{12}
\end{aligned}
$$

where $\Delta \phi=\phi^{\prime}-\phi$.

Finally, when the scattering occurs out of resonance, the density matrix of the scattered photon is given by the Equation (4) of Ahn et al. (2002). This equation is also rearranged as follows:

$$
\begin{aligned}
\rho_{11}^{\prime}= & \left(\cos ^{2} \Delta \phi\right) \rho_{11} \\
- & (\cos \theta \sin 2 \Delta \phi) \rho_{12} \\
+ & \left(\sin ^{2} \Delta \phi \cos ^{2} \theta\right) \rho_{22} \\
\rho_{12}^{\prime}= & \left(\frac{1}{2} \cos \theta^{\prime} \sin 2 \Delta \phi\right) \rho_{11} \\
+ & \left(\cos \theta \cos \theta^{\prime} \cos 2 \Delta \phi+\sin \theta \sin \theta^{\prime} \cos \Delta \phi\right) \rho_{12} \\
+ & \cos \theta\left(-\sin \theta \sin \theta^{\prime} \sin \Delta \phi\right. \\
& \left.-\frac{1}{2} \cos \theta \cos \theta^{\prime} \sin 2 \Delta \phi\right) \rho_{22} \\
= & \left(\cos \theta^{2} \sin { }^{2} \Delta \phi\right) \rho_{11} \\
\rho_{22}^{\prime} & \cos \theta^{\prime}\left(2 \sin \theta \sin \theta^{\prime} \sin \Delta \phi\right. \\
& \left.+\cos \theta \cos \theta^{\prime} \sin 2 \Delta \phi\right) \rho_{12} \\
+ & \left(\cos \theta \cos \theta^{\prime} \cos \Delta \phi+\sin \theta \sin \theta^{\prime}\right)^{2} \rho_{22} .
\end{aligned}
$$

The angular distribution of the scattered radiation for an incident photon with $\hat{\mathbf{k}}$ and $\rho(\theta, \phi)$ is given by the trace of the density operator $\rho^{\prime}\left(\theta^{\prime}, \phi^{\prime} ; \theta, \phi\right)$ (e.g. Lee $\&$ Blandford 1997). Therefore, once the wavevector $\hat{\mathbf{k}}^{\prime}$ of a scattered photon is chosen from $\rho^{\prime}$ in accordance with the scattering type, the polarization state is also determined at the same time. It is noted that the degree of polarization is computed only after the new density matrix is normalized so that it has a unit trace.

\section{Results}

\subsection{Degree of Linear Polarization}

We compute the degree of linear polarization of $\mathrm{Ly} \alpha$ photons emergent from an optically thick slab with unpolarized sources distributed in the central plane. In Figure 2, we show the degree of linear polarization for various optical depths according to $\mu$ that represents the observer's line of sight with respect to the slab normal. In Figure 2, we fix the Voigt parameter $a=1.49 \times 10^{-2}$ and consider slabs with line center scattering optical depths of $\tau_{0}=2 \times 10^{4}, 5 \times 10^{4}, 2 \times$ $10^{5}, 5 \times 10^{5}$, and $2 \times 10^{6}$. Therefore, the slabs considered in this calculation are characterized by the values of $a \tau_{0}=3 \times 10^{2}, 7.5 \times 10^{2}, 3 \times 10^{3}$, and $7.5 \times 10^{3}$, respectively. In the figure, the dots with $1 \sigma$ error bars represent our Monte Carlo results and the solid lines represent the result for Thomson-scattered continuum photons transferred in a semi-infinite electron cloud (e.g., Chandrasekhar 1960, Angel 1969, Phillips \& Mészáros 1986). It is noticeable that the degree of Ly $\alpha$ polarization for $a \tau_{0}>10^{3}$ is very similar to that of the Thomson-scattered radiation in a thick slab.

In a slab with $a \tau_{0}>10^{3}$, the Ly $\alpha$ transfer processes are characterized by the spatial transfer associated with a number of successive wing scatterings that Ly $\alpha$ photons experience just before escape (Ahn et al. 2002). 


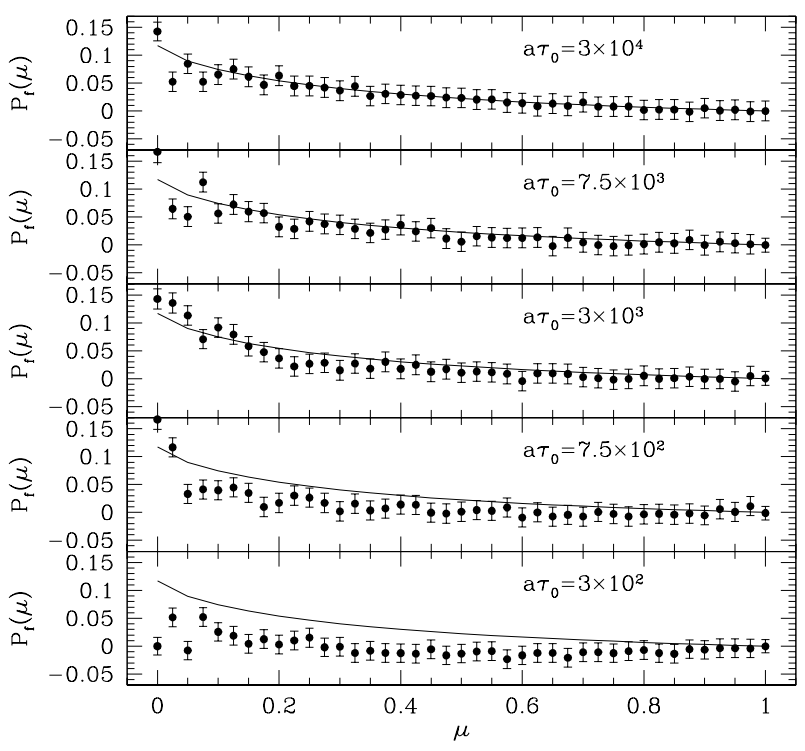

Figure 2. Polarization of the Ly $\alpha$ photons, summed over frequencies, emergent from the H I medium with an unpolarized source located at the midplane of the slab as illustrated in Figure 1. We show five cases with different $a \tau_{0}$. The solid line in each box represents the limiting behavior for the Thomson scattering.

The characteristic number of last successive wing scatterings is determined by the 'wing scattering optical depth', $\tau_{w}$ at the characteristic frequency of the emergent photons, given approximately by

$$
\tau_{w}=\frac{1}{\sqrt{\pi}} x_{s}
$$

where $x_{s}=\left(a \tau_{0}\right)^{1 / 3}$. According to Ahn et al. (2002), this is valid only for $a \tau_{0} \geq 10^{3}$, when the diffusion approximation can be safely applied.

In the cases considered in Figure 2, the characteristic wing optical depths are $\tau_{w}=3.8,5.1,8.1,11.0$, and 17.5, respectively. According to Phillips \& Mészáros (1986), the polarization of the Thomson scattered radiation emerging from a finitely thick slab with $\tau_{e} \geq 10$ is almost indistinguishable from that of the radiation emerging from a slab with infinite optical depth. In a similar way, the polarization of $\operatorname{Ly} \alpha$ exhibits the same limiting behavior to that of the Thomson scattered radiation when $a \tau_{0} \geq 10^{3}$ or $\tau_{w} \geq 10$. This behavior lends strong support to the interpretation that the Ly $\alpha$ transfer is characterized by the successive wing scatterings just before escape when $\tau_{w}$ is fairly large.

\subsection{Spectropolarimetry of $\mathrm{Ly} \alpha$}

In this subsection we discuss spectropolarimetric observations for Ly $\alpha$ emitting objects. In Figures 3-7 we show our results of the Monte Carlo calculations for the linear polarization of $\operatorname{Ly} \alpha$ photons emerging from an extremely thick hydrogen slab, where the polarization is shown as a function of relative frequency

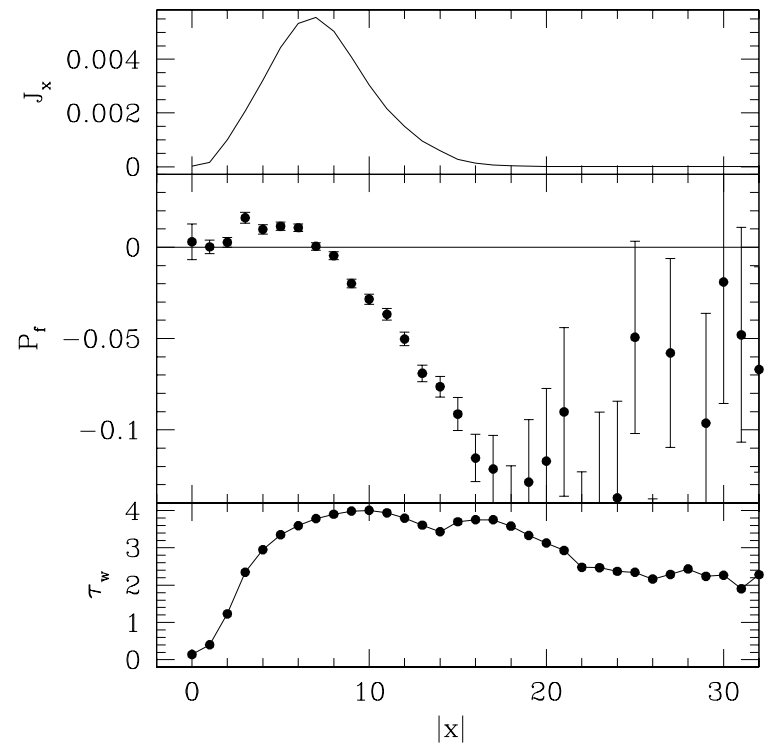

Figure 3. Simulated spectropolarimetry of Ly $\alpha$ emission for the unpolarized source at the central plane of a slab with the vertical optical depth from the center of the plane $\tau_{0}=$ $2 \times 10^{4}$ and the Voigt parameter $a=1.49 \times 10^{-2}$. The top panel shows the emergent flux, the middle panel shows the degree of linear polarization, and the bottom panel shows the effective wing optical depth $\tau_{w}=\sqrt{N_{w}}$ where $N_{w}$ is the number of successive wing scatterings just before escape. All the quantities shown in the figure have been obtained after averaging over all emergent angles $\theta=\cos ^{-1} \mu$.

shift from the line center. The H I slabs considered in Figures 3-7 have normal scattering optical depths of $\tau_{0}=2 \times 10^{4}, 5 \times 10^{4}, 2 \times 10^{5}, 5 \times 10^{5}$, and $2 \times 10^{6}$, as in Figure 2. The total number of photons used in each simulation is $1.6 \times 10^{6}$, and the relative frequency bin is set to $\Delta x=1$. An exception is made for $\tau_{0}=2 \times 10^{6}$, in which we set $\Delta x=2$. All the quantities shown in the figures have been obtained after averaging over all emergent angles $\theta=\cos ^{-1} \mu$.

In the top panels of Figures 3-7, we show the emergent fluxes, in the middle panel the degree of linear polarization $P_{f}$, and in the bottom panel the characteristic wing scattering optical depth $\tau_{w}$. Treating the radiative transfer in a Thomson scattering medium as a random walk process, we inspect $N_{w}$, the average number of successive wing scatterings just before escape, to calculate the characteristic wing scattering optical depth $\tau_{w}=\sqrt{N_{w}}$.

It is immediately seen that negligible polarization develops near the line center. This is because photons with frequencies near the line-center escape through a single longest flight after a large number of core scatterings near the surface, where the local core scatterings isotropize the radiation field. The core-wing boundary frequency is approximately given by Equation (13) with $\tau_{w}=1$, or $x=\sqrt{\pi}$. Hence our results in Figures $3-7$ show that $P_{f}=0$ in the frequency interval $0 \leq x \leq \sqrt{\pi}$.

Another point is that the photons filling in the near 


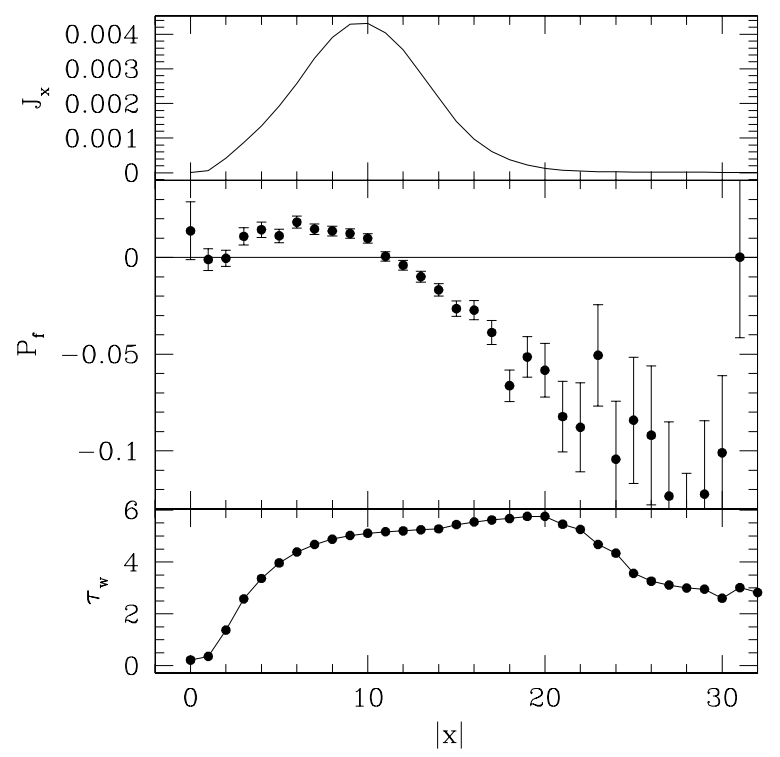

Figure 4. Same as Figure 3, for $\tau_{0}=5 \times 10^{4}$.

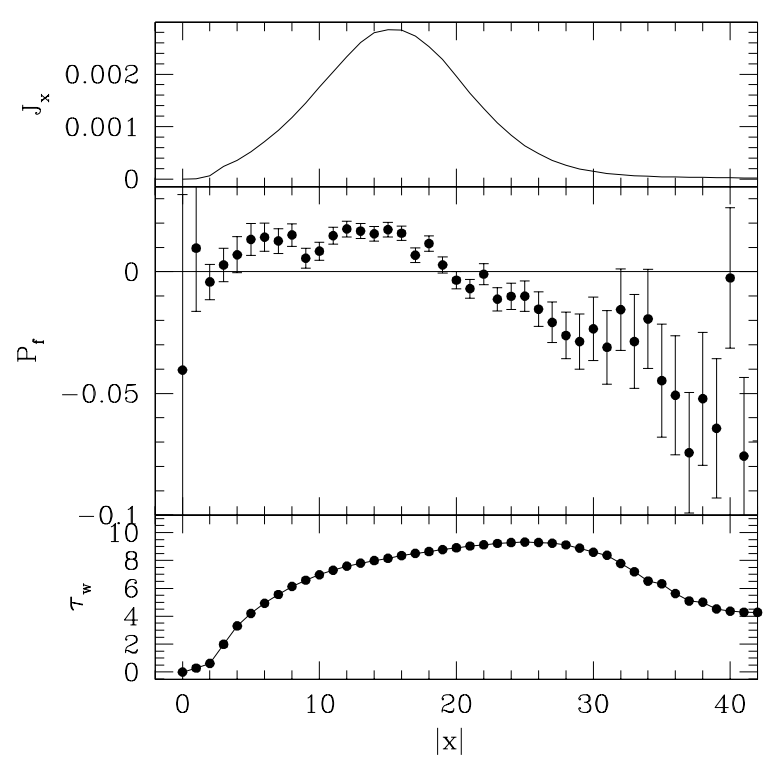

Figure 5. Same as Figure 3, for $\tau_{0}=2 \times 10^{5}$.

wing part of $\operatorname{Ly} \alpha$ emission are positively polarized, which means the electric vectors of those escaping photons tend to lie in the direction perpendicular to the slab-normal, i.e., parallel to the slab. This can be compared with the case in Phillips \& Mészáros (1986) for the grazing direction with large optical depth. In this study, the degree of linear polarization in this part is approximately 1.5 percent, which is significantly smaller than the maximum value of 11.7 percent given by Chandrasekhar (1960) and Phillips \& Mészáros (1986). The smaller polarization in our study is found because we average polarization over emergent angles. We inspect the number of successive wing scatterings just before escape and calculate the corresponding wing scattering

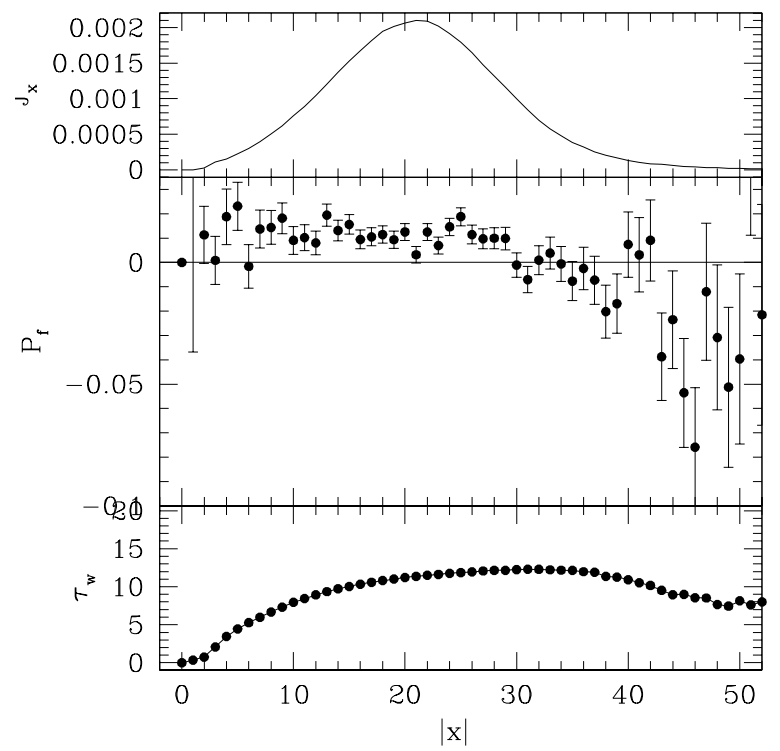

Figure 6. Same as Figure 3, for $\tau_{0}=5 \times 10^{5}$.

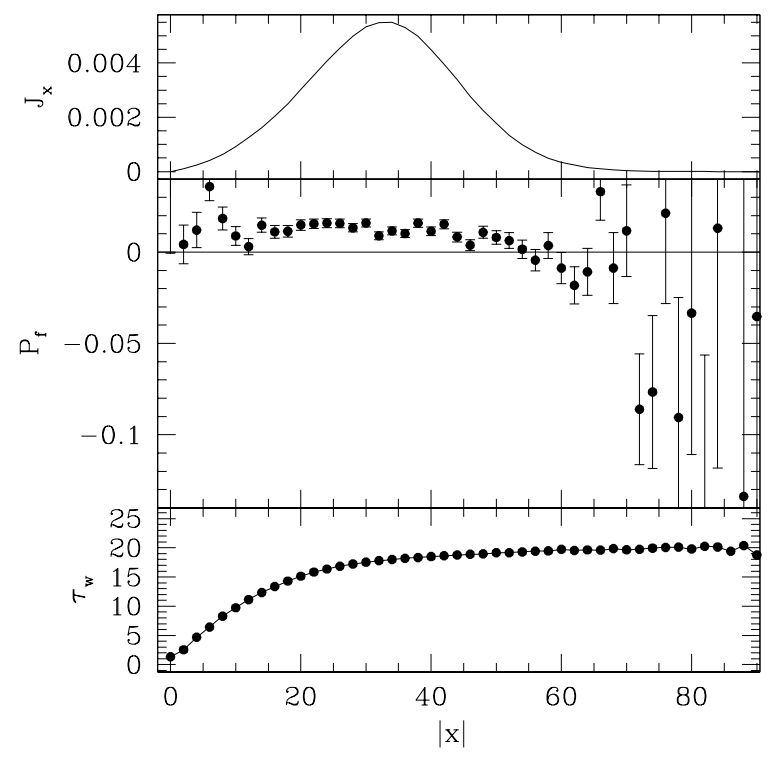

Figure 7. Same as Figure 3, for $\tau_{0}=2 \times 10^{6}$.

optical depths. We show the results in the lower panels of Figures 3-7. These results show that the near wing part of the emergent profiles is contributed by photons with large wing scattering numbers. Therefore, a large number of wing scatterings accompanied by spatial transfer preferentially in the normal direction may cause the electric vectors of the line photons to get aligned in the direction perpendicular to the slab normal, i.e., to the direction parallel to the slab.

It is also notable that the degree of linear polarization becomes negative at far wing frequencies, indicating that the polarization direction flips from the perpendicular direction to the parallel direction to the slabnormal as the frequency-shift $\Delta x$ from the line-center 
increases. The polarization in the far-wing part can also be understood by comparison with the results given in Phillips \& Mészáros (1986) for the optically thin cases.

Another point we can observe in Figures 3-7 is that the strength of the negative polarization in the far wing regime decreases as $a \tau_{0}$ increases. This effect can be understood in terms of the anisotropic angular distribution of the emergent Ly $\alpha$, which was called the 'beaming effect' by Ahn et al. (2002). They investigated the beaming of Ly $\alpha$ photons for the cases of very large $a \tau_{0}$, in which most photons escape from the extremely thick slab-like media favorably in the direction normal to the slab plane. This is also the case in an optically thick Thomson-scattering cloud (Phillips \& Mészáros 1986). The fraction of Ly $\alpha$ photons emergent in the direction parallel to the slab surface decrease as $a \tau_{0}$ increases. As a result, their contribution to negative polarization diminishes, and eventually the degree of linear polarization in the far wing parts becomes larger and eventually positive. Due to the beaming effect, the polarization flip occurs farther away from the line-center as $a \tau_{0}$ increases. The anisotropy in angular distribution will be discussed in more detail in the next subsection.

The polarization of Ly $\alpha$ photons in an anisotropically expanding slab was investigated by Lee \& Ahn (1998). Their calculation for the cases with the line center optical depth $\tau_{0} \leq 10^{5}$ and the Voigt parameter $a=4.71 \times 10^{-4}$ shows that the polarization flip occurs near the line center, leading to the emergent peaks that are negatively polarized on average. Because the bulk motion of the expanding slab enhances the escape of line photons, the polarization flip occurs at smaller optical depths than in the static medium with the same neutral hydrogen column density.

\subsection{Angular distribution of the polarized $\operatorname{Ly} \alpha$ flux}

In this subsection, we investigate the angular distribution of the emergent Ly $\alpha$. Figure 8 of Ahn et al. (2002) shows the frequency-integrated specific intensity $I(\mu) \equiv F(\mu) / \mu$, where $F(\mu)$ is the frequency-integrated flux at angle $\mu$. Ahn et al. (2002) discovered that the Ly $\alpha$ limb brightening occurs when $a \tau_{0} \ll 10^{3}$, and that the Ly $\alpha$ limb darkening appears when $a \tau_{0}>10^{3}$. Here in this subsection, we show the polarization $\left(P_{t}\right)$ of the total emergent flux. This quantity is obtained from the definition $P_{t}=\int P_{f}(\mu) I(\mu) \mu d \mu$, where $P_{f}(\mu)$ is shown in Figure 2 and $I(\mu)$ is shown in Figure 8 of Ahn et al. (2002).

Figure 8 shows the degree of linear polarization as a function of $a \tau_{0}$. Here $a \tau_{0}$ can be thought to be a measure of relative importance between wing scatterings and core scatterings in the line transfer. The error in $P_{t}$ is simply propagated from $P_{f}(\mu)$. The most important thing to note in Figure 8 is that the polarization sign changes as $a \tau_{0}$. For $a \tau_{0}<750$, the polarization direction is parallel to the slab normal, while for $a \tau_{0}>750$ it is perpendicular to it.

As we saw in Section 3.1, the case with $\tau_{0}=2 \times 10^{4}$ and $a=1.49 \times 10^{-2}$ has the characteristic optical depth $\tau_{w}=3.8$. We can understand the properties

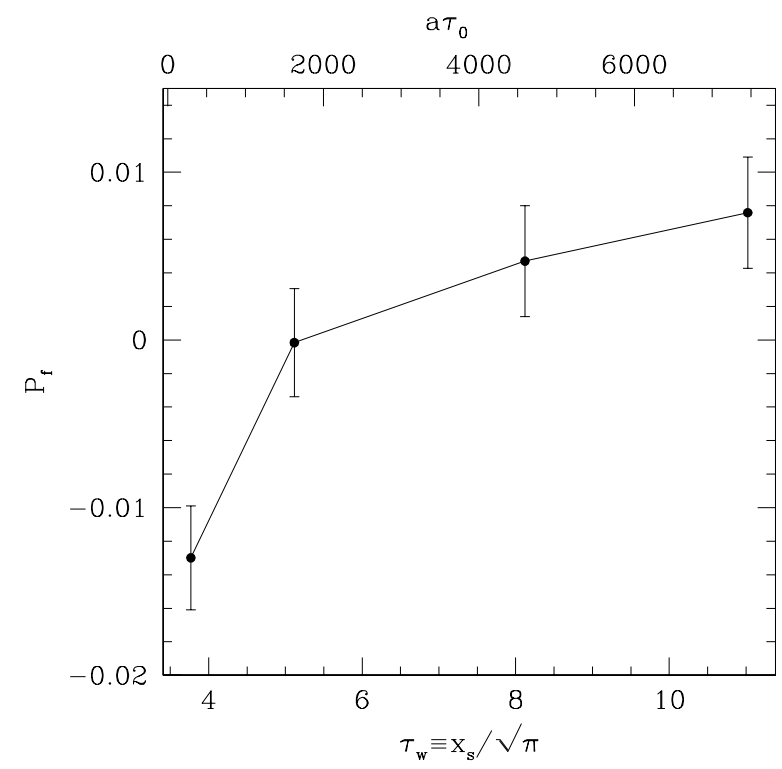

Figure 8. Degree of linear polarization $\left(P_{f}\right)$ averaged over both frequency and angular distribution. We show the results for various optical depths and Voigt parameters. $P_{f}$ has the negative value for $a \tau_{0}<10^{3}$, while positive values are obtained for $a \tau_{0}>10^{3}$.

of Ly $\alpha$ wing scatterings by comparing with the Thomson scattering in an optically thick electron cloud with $\tau_{e} \simeq \tau_{w}$, because the scattering is described by the same Rayleigh phase function and spatial diffusion dominates the transfer process.

A close examination of the curves in Figure 2 and Figure 5 in Phillips \& Mészáros (1986) and those in Figure 2 and Figure 8 in this work shows that when $a \tau_{0}<10^{3}$ the photon flux emergent in the grazing direction of the slab surface increases as $a \tau_{0}$ decreases. On the other hand, the beaming of emergent Ly $\alpha$ is enhanced as $a \tau_{0}$ increases. As a result, the critical frequency of the polarization flip moves toward the larger value, which we can see in Figures 3-7.

\section{Conclusions}

We have investigated the linear polarization of $\mathrm{Ly} \alpha$ emergent from a static and uniform $\mathrm{H}$ I slab with a scattering optical depth that ranges from moderately large to extremely huge. The polarization behavior of the Ly $\alpha$ can be understood qualitatively by comparing with that of the Thomson scattered radiation. In particular, the polarization behavior is mainly determined by the Voigt parameter $a \tau_{0}$. When the scattering medium is extremely thick with $a \tau_{0}>10^{3}$, Ly $\alpha$ photons emerging in the direction perpendicular to the slab normal are polarized parallel to the slab plane with the degree up to 12 percent, which is the same value as that of the Thomson scattered radiation in a semi-infinite thick medium (Chandrasekhar 1960).

The variation of linear polarization with $a \tau_{0}$ also shows similar behavior with that of the Thomson scattered radiation (Phillips \& Mészáros 1986). The linear polarization of Ly $\alpha$ develops in the direction perpendic- 
ular to the slab plane when $a \tau_{0}<10^{3}$, while it flips to become parallel to the slab plane when $a \tau_{0}>10^{3}$. Our simulated spectropolarimetry of Ly $\alpha$ shows the polarization flip in the spectra. The linear polarization near the line center is almost zero. The wing parts near the line center are polarized in the direction perpendicular to the slab normal, while the far wing parts are polarized in the direction parallel to the slab normal. The zero polarization around the resonance frequency is caused by resonance scatterings just before escape which isotropize the electric vector of the Ly $\alpha$ photons. The perpendicular polarization at the far wing parts is caused by Ly $\alpha$ photons preferentially emerging in the grazing direction. The Ly $\alpha$ photons constituting the near wing part of the emergent peaks have large wing optical depths, and therefore they are beamed in the slab normal direction, which gives rise to positive polarization. As $a \tau_{0}$ increases, the beaming effect prevents Ly $\alpha$ photons with far wing frequencies from emerging into the grazing direction. Therefore, the location of the polarization flip in the Ly $\alpha$ line can be an important indicator of the value of $a \tau_{0}$.

\section{ACKNOWLEDGMENTS}

This research was supported by the Basic Science Research Program through the National Research Foundation (NRF-2014R1A1A2054887).

\section{REFERENCES}

Adams, T. 1972, The Escape of Resonance-Line Radiation from Extremely Opaque Media, ApJ, 174, 439

Ahn, S.-H., Lee, H.-W., \& Lee, H. M. 2000, Ly Alpha Transfer in a Thick, Dusty, and Static Medium, JKAS, 33, 29

Ahn, S.-H., Lee, H.-W., \& Lee, H. M. 2001, Ly $\alpha$ Line Formation in Starbursting Galaxies. I. Moderately Thick, Dustless, and Static H I Media, ApJ, 554, 604

Ahn, S.-H., Lee, H.-W., \& Lee, H. M. 2002, Ly $\alpha$ Line Formation in Starbursting Galaxies. II. Extremely Thick, Dustless, and Static H i Media, ApJ, 567, 922

Ahn, S.-H., Lee, H.-W., \& Lee, H. M. 2003, P Cygni Type Ly $\alpha$ from Starburst Galaxies, MNRAS, 340, 863
Angel, J. R. P. 1969, Polarization of Thermal X-Ray Sources, ApJ, 158, 219

Avery, L. W., \& House, L. L. 1968, An Investigation of Resonance-Line Scattering by the Monte Carlo Technique, ApJ, 152, 493

Bouwens, R. J., Thompson, R. I., Illingworth, G. D., et al.

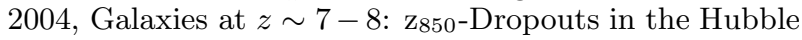
Ultra Deep Field, ApJ, 616, L79

Chandrasekhar, S. 1960, Radiative Transfer (New York: Dover)

Harrington, J. P. 1973, The Scattering of Resonance-Line Radiation in the Limit of Large Optical Depth, MNRAS, 162, L43

Hayes, M., Scarlata, C., \& Siana, B. 2011, Central Powering of the Largest Lyman- $\alpha$ Nebula is Revealed by Polarized Radiation, Nature, 476, 304

Kunth, D., Mas-Hesse, J. M., Terlevich, E., et al. 1998, HST Study of Lyman-alpha Emission in Star-Forming Galaxies: the Effect of Neutral Gas Flows, A\&A, 334, 11

Lee, H.-W.,\& Ahn, S.-H. 1998, Polarization of the Ly $\alpha$ Line from an Anisotropically Expanding H I Shell in Primeval Galaxies, ApJ, 504, L61

Lee, H.-W., \& Blandford, R. D. 1997, On the Polarization of Resonantly Scattered Emission Lines - III. Polarization of Quasar Broad Emission Lines and Broad Absorption Line Troughs, MNRAS, 288, 19

Lee, H.-W., Blandford, R. D., \& Western, L. 1994, On the Polarization of Resonantly Scattered Emission Lines - I. Emission and Absorption Coefficients in an Anisotropic Radiation Field, MNRAS, 267, 303

Neufeld, D. A. 1990, The Transfer of Resonance-Line Radiation in Static Astrophysical Media, ApJ, 350, 216

Pettini, M., Shapley, A. E., Steidel, C. C., et al. 2001, The Rest-Frame Optical Spectra of Lyman Break Galaxies: Star Formation, Extinction, Abundances, and Kinematics, ApJ, 554, 981

Phillips, K. C., \& Mészáros, P. 1986, Polarization and Beaming of Accretion Disk Radiation, ApJ, 310, 284

Stenflo, J. O. 1980, Resonance-Line Polarization. V Quantum-Mechanical Interference between States of Different Total Angular Momentum, A\&A, 84, 68

Stenflo, J. O. 1994, Solar Magnetic Fields - Polarized Radiation Diagnostics (Dordrecht: Kluwer)

Stenflo, J. O. 1996, Scattering Physics, Solar Phys., 164, 1 